\section{Androgens and Diversity in Adult Human Partnering}

\author{
Sari M. van Anders
}

\section{Introduction}

In this chapter, I survey testosterone $(\mathrm{T})$ and diversity in adult human partnering, with attention to considerations about diversity and how various empirical findings bear on evolutionary understandings of partnering, sexual orientation, and life strategies. I begin by asking and attempting to answer the question, Why diversity? As diversity in human partnering is tied to sexual orientation in many ways, I next review research that examines whether and how prenatal and circulating hormones may be associated with sexual orientation in women and men. I then present a brief review of research on androgens and partnering in men (see Gray and Campbell, this volume, for a more comprehensive review) and move into discussions of theoretical frameworks for conceptualizing T-partnering associations. I subsequently explore contributions to our understanding of partnering and hormones from research that incorporates diverse populations, including research that addresses gender, sexual orientation, and relationship type. I close with a summary that emphasizes the value of diversity to behavioral neuroendocrine understandings of partnering.

Research into social relationships and hormones is grounded in evolutionary theory and can be conducted using a variety of levels of analysis. In humans, this generally has translated into behavioral and endocrine analyses, with a focus on ultimate and proximate mechanisms. At the proximate level, we can ask: Do hormones affect partnering? Does partnering affect hormones? At the ultimate level, we can ask: Why would hormones affect partnering, or partnering hormones, and how might this be adaptive? While theorizing at the ultimate level can guide our empirical research at the proximate level in terms of hypothesis generation, understanding the proximate mechanisms is really a prerequisite to proposing ultimate explanations. While this may seem tautological to some, it is no less a derivative of scientific method than in any other field, with its reliance on theory-derived hypotheses that are tested empirically, leading to evidence for or against a theoretical position and resulting in revised or strengthened theory.

\section{Why Diversity?}

I use the term diversity to refer to the broad spectrum of partnering styles and behaviors that are present in humans. Diversity is often used euphemistically when referring to people and is most often understood to imply sexual minorities, women, ethnic minorities or any minority, underrepresented, or "othered" groups. In contrast, diversity is a foundational aspect of behavioral neuroendocrine research, and the term is employed to refer to the naturally occurring broad spectrum of behaviors, phenotypes, and strategies apparent throughout the animal kingdom. Quick perusals of behavioral neuroendocrine textbooks (for example, Becker et al., 2002; Nelson, 2005) attest to the striking variety in gender morphs, sexual behaviors, and sexual differentiation under study. Without this naturally occurring diversity, behavioral neuroendocrinology would arguably lose much of its content and unquestionably lose a great deal of its most fascinating subject matter.

The study of partnering and hormones has generally focused on the diversity of pair-bonding apparent within and between animal species as well as heterosexual men in both North American and international populations. Additionally, researchers have long focused on clinical populations with conditions affecting their endocrine function or circulating hormones to address endocrine questions that cannot be answered in humans exposed to typical endogenous hormones. There is an additional approach to addressing those endocrine questions, and that is to look to the naturally occurring diversity in human relationship styles. Just as zoologists and ecologists have learned from the stunning variety of behavioral phenotypes, a creative and resourceful approach to human diversity should lead to some answers and more questions about partnering and hormones. Why not, occasionally, use "human models" for human research?

Research has largely focused on men, possibly because major theoretical perspectives focus on male-male competition for mates and male 
paternal/mate investment. Another possible reason is the large number of women who are either pregnant, postmenopausal, lactating, or using hormonal contraceptives and who therefore have altered endogenous endocrine profiles that can confound research and limit the potential pool of women participants. A third possible reason is that women show menstrual variation in hormones in addition to the seasonal (Dabbs, 1990b; Wisniewski and Nelson, 2000; van Anders, Hampson, and Watson, 2006) and diurnal (Rose et al., 1972) patterns evidenced by both women and men. A fourth reason might be limited access to women in international research. A fifth possible reason is that the field is relatively new, and testing men has just come before testing women. Additionally, researchers interested in androgens often focus on males (but see Ketterson, Nolan, and Sandell, 2005). However, as articulated elsewhere (van Anders and Watson, 2006b), including women is really not that difficult and is largely warranted by theoretical considerations.

If women have not been included, it should not be surprising that the focus has not been merely on men but on heterosexual men or at least men in opposite-sex pairings. Again, there are several possible reasons. There is a relatively small number of nonheterosexual individuals in society. Also, implicit theoretical positions that focus on male-female pair bonds because of their reproductive potential might limit the scope. Related might be the continuing view of same-sex sexual orientations as evolutionarily paradoxical, with the unresolved (though arguably constructed) paradox being that same-sex sexual orientation appears to be somewhat "biological" in origin for at least some people but is theorized by some to lead to decreased reproduction (and fitness). Other possibilities include discomfort with including a minority group for reasons of personal bias that could be of a prejudicial origin or of a wish to "leave alone" a group that has not always benefited from scientific attention. Inclusion of nonheterosexual groups is, however, crucial to understanding human partnering and hormones for a variety of reasons that I detail later and briefly allude to here. There are a consistent and significant number of people in same-sex relationships, and so to understand human partnering and hormones in entirety, we should include the entirety of the human experience (though, maybe, not all in one study). Including more than one mode of partnering is likely to shed light not only on same- and opposite-sex partnering and hormones but on broad understandings of partnering and hormones, providing insights that could not be gleaned otherwise.

There are a variety of diverse relationship styles that could be included in research in adult partnering and hormones, and these could be deemed human models, though it might be apparent how little any person or group would appreciate being so labeled or perceived. Humans have sometimes been described as serially monogamous, but some people stay with only one person. Some people have multiple partners simultaneously-or would if they could find willing individuals or accepting social structures. Some people live with their partners; some live apart. Some partner for love, some partner for loneliness avoidance, and some partner for family concerns. There is variety and diversity in how and why adults partner that can be related to choice, accident, or opportunity, affording us myriad relationship statuses, styles, and desires to study. Someone who longs for a long-term, committed relationship with one person is qualitatively different from someone who has little interest in commitment, at least in terms of commitment desire. Do they differ in other ways? Do they differ in endocrine parameters?

Including groups in research necessitates looking for them, and looking for them necessitates knowing that they exist and postulating that they are relevant to the topic at hand. However, some linguistic conventions may obscure forms of partnering that are less visible than heterosexual pair-bonds but important for understanding how partnering and hormones are associated. For example, mating relationship may seem like an appropriate descriptor stemming from nonhuman research, but not all relationship types are covered by this terminology. Though mate can refer to a spouse, counterpart, or one of a pair, I think it connotes a reproductive partner when used in biologically oriented research. Just as empirical studies show that man brings images of men to mind, and not humans more generally or women (Crawford, 2001), I think that mate brings reproductive partners to mind, even when users have less specific intentions, and therefore obscures forms of relationships that we do not intentionally mean to exclude.

These may seem like semantic quibbles, but semantics can predispose us to focus on certain types of relationships, and this can prevent us from including populations that are likely to be helpful in understanding how hormones and partnering are associated. Thus semantics (or not attending to them) can impede scientific progress. How can this be addressed? One possibility is to use mate expansively, since English is an infamously flexible language. Another option is to use more inclusive terminology. In this chapter, I use partnering, which has a similar meaning to mating but does not exclusively denote reproductive relationships.

Conducting scientific research with groups who are less visible brings its own challenges and considerations, It is impossible for experimenter to consider the inclusion of a group of which they have no awareness. 
Potentially most useful are open-ended questions on questionnaires in ongoing studies. Participants can then write in their own responses, which can alert the researcher to linguistic conventions that indicate unknown (to the scientist) groupings. Similarly, taking participants' comments as helpful and potentially "expert" input (for example, "I don't fit on your questionnaire, and I think you should include this other category") can be instructive. Allowing for verbal or written feedback can also be helpful. Finally, at this early stage, sensitivity in terminology and communications regarding the study and group should go far toward reducing unintended and undesired negative repercussions.

I will focus on literature that reflects diversity in associations between androgens and partnering, including relationship status and relationship "orientation." Most people are more familiar with sexual orientation as a topic of scientific inquiry than with relationship status or orientation, as media reports of biological bases of sexual orientation are widely dispersed and attended to. Diversity in relation to androgens and partnering likely brings sexual orientation to mind, and this more established line of inquiry is an appropriate starting point for a discussion of hormones and partnering in humans.

\section{Androgens and Sexual Orientation}

One additional feature of attraction that is an important determinant of relationships is sexual orientation. Sexual orientation has been defined by some as a "dispositional sexual attraction towards persons of the opposite sex or same sex" (Rahman, 2005b, 1057) but is understood by others to reflect a less tangible and more contextualized concept (for example, L. Diamond, 2003). Certainly, scientists and laypeople alike use the term to refer to the direction of a person's attraction to same- or opposite-sexed individuals in conjunction with their desire to partner with these individuals and identify as someone who partners with these individuals. Sexual orientation has largely come to replace sexual preference, reflecting a shift in perception that the construct is a trait with at least some biological/innate causation.

While sexual orientation has come to connote a somewhat fixed and biological predisposition to same- or opposite-sex individuals (not to reify sex boundaries), researchers have studied what links exist between biology and sexual orientation (for example, Rahman, 2005b). Hormones are attended to, stemming from the idea that a gay/lesbian sexual orientation represents a sex-atypical orientation and thus sex-atypical sex hormone exposure (see Gorman, 1994, for an appropriately critical discussion of this approach). This line of theorizing is based on animal literature, where exposure to "cross-sex" hormones can affect sexual behaviors and interests toward same-sexed animals (for example, Baum, 2006).

Researchers generally divide endocrine effects into those that are organizational and those that are activational. In humans, organizational effects are generally prenatal and permanent and have a "hard-wiring" effect (for more on organizational verses activational effects, see Wallen and Hassett, this volume; McIntyre and Hooven, this volume). The prenatal androgen hypothesis has thus been proposed as an explanation for the development of same-sex sexual orientations. The hypothesis holds that higher-than-typical prenatal androgens predispose women to be sexually oriented toward women, and lower-than-typical prenatal androgens predispose men to be sexually oriented toward men.

\section{Prenatal Hormones and Sexual Orientation}

MEASURING PRENATAL HORMONES

Understanding the contributions of prenatal hormones in humans to any aspect of behavior is challenging because we cannot reliably measure prenatal hormones in random samples of people. Amniocentesis is one way to measure prenatal hormones, but the procedure leads to an increased risk of spontaneous abortion, limiting its use to clinical populations of women for whom the benefits (for example, potentially discovering a serious medical condition) outweigh the risks. Because amniocentesis is performed at varying points during pregnancy, gestational age and associated variation in $\mathrm{T}$ are difficult to control, leading to extreme variability and often difficulties of interpretation.

Maternal hormones have also been used as potential measures of fetal endocrine exposure and could theoretically represent maternal-plus-infant circulating hormones, but the broad changes and fluctuations in maternal hormones over gestation limit this approach. However, there is something of a consensus that this approach is not well supported (for example, Cohen-Bendahan et al., 2005). Some researchers have assayed hormones from umbilical cords, and this could be useful, though there are obvious caveats. Umbilical cord measures likely represent circulating hormones at time of parturition (when hormones are askew already). And umbilical cords link the fetus and mother, so cord measurements thus reflect both maternal and fetal hormonal contributions (for a review of these methods, see Cohen-Bendahan, van de Beek, and Berenbaum, 2005).

An additional approach is to include people who have clinical conditions affecting their hormone release, either prenatally (that is, organizationally) 
or postnatally (that is, activationally). Several conditions have been identified, with those affecting prenatal hormones receiving more attention. In congenital adrenal hyperplasia (CAH), cortisol cannot be produced because of a missing or faulty enzyme that converts precursors to cortisol. As a result, the fetal pituitary releases increasing amounts of adrenocorticotropic hormone (ACTH) in an attempt to increase cortisol production in the adrenal cortex. Though cortisol cannot be made or released, other adrenal hormones-like androgens-can. As a result, fetuses with $\mathrm{CAH}$ are exposed to higher-than-typical androgens, and females can be born with genitals showing various degrees of virilization. Androgen insensitivity syndrome (AIS) has also received attention; in this genetic condition, $\mathrm{XY}$ individuals have androgen receptors that are nonfunctional (complete AIS) or only partially functional (partial AIS). Androgen levels are high in people with AIS but exert no or partial effect because of the nonfunctional or faulty androgen receptors. As a result, fetuses with complete AIS are not exposed to androgens (despite high circulating levels) and are born with female-typical genitals, and fetuses with partial AIS are exposed to inconsistent amounts of androgens and are born with genitals that show various degrees of virilization. In XY individuals, another condition called 5-alphareductase deficiency can be present, and since the 5 -alpha-reductase enzyme converts $\mathrm{T}$ to the more potent dihydro $\mathrm{T}$ (which mediates genital virilization), individuals are born with female-appearing genitals. Individuals with other clinical conditions have also received attention (for a review, see Gooren, 2006), as have people exposed to exogenous hormones prenatally. For example, diethylstilbestrol (DES) is a potent synthetic estrogen that has masculinizing effects and was administered to pregnant women.

Research on sexual orientation and hormones via clinical conditions has been valuable to scientists (though not overly helpful or welcome to the individuals themselves), but there is the possibility that findings are not generalizable beyond clinical populations to adults exposed to typical hormones. It is unclear whether findings from clinical populations exposed to higher- or lower-than-typical hormones can be generalized to healthy populations of adults exposed to hormones that are high or low but still in the typical range. As well, populations with relevant endocrine conditions are not large, limiting sample size.

Psychologists have thus also turned to potential markers of fetal endocrine exposure, and generally any variable showing sex differences has been hypothesized to be associated with prenatal androgens, for example, otoacoustic emissions (OAEs). OAFs are quict clicks made by the inner ear. They can occur naturally or in response to external sounds and are more frequent in females, with evidence supporting prenatal andro gen masculinization effects (for example, McFadden, 1993; McFadden Loehlin, and Pasanen, 1996). Another measure is digit ratio, which is a ratio of the length of the second to fourth digit (that is, finger). Digit ratios show sexual dimorphism, with male ratios lower than females (Manning et al., 1998), and there is some evidence that digit ratios are associated with prenatal androgens (Brown, Hines, et al., 2002; Lutchmaya et al., 2004; van Anders, Wilbur, and Vernon, 2006), though this putative association is still controversial. The theoretical grounding for such an association is that fingers develop under the same genetic control as external genitals (HoxD and HoxA: Kondo et al., 1997) with coordinated expression (Peichel, Prabhakaran, and Vogt, 1997). They thus develop at the same time, when prenatal androgen levels are high to promote sexual differentiation and masculinization of bipotential gonads. Whether the digits respond to androgens has not been examined (for example, whether they are rich in androgen receptors).

PRENATAL HORMONES AND SEXUAL ORIENTATION IN WOMEN

Studies of possible effects of prenatal hormones on sexual orientation in women have often focused on women with CAH. There is some evidence that women with $\mathrm{CAH}$ are more likely to report same-sex sexual fan tasies and behavior (Dittmann et al., 1992) or less opposite-sex sexual fantasy and behavior (Zucker et al., 1996; Hines, Brook, and Conway, 2004 ) than unaffected controls. Similarly, women exposed to exogenous hormones like DES administered during their mother's pregnancies show slight increases in same-sex sexual orientation in addition to affected genital development (Ehrhardt et al., 1985; Meyer-Bahlburg et al., 1985). As well, women with complete androgen insensitivity syndrome (CAIS) who are exposed to no circulating androgens show no difference in sexual orientation compared to unaffected control women (Wisniewski et al., 2000; Hines, Ahmed, and Hughes, 2003).

Using putative markers of prenatal androgens, women with same-sex sexual orientations tend to have lower (that is, more male-typical) digit ratios than women with opposite-sex sexual orientations (Williams et al., 2000; Rahman and Wilson, 2003; Rahman, 2005a; compare Lippa, 2003; van Anders and Hampson, 2005). And there is some evidence that gendered variation within women might be associated with prenatal an drogens. For example, Brown, Finn, et al. (2002) found that digit ratios were masculinized in self-identified butch lesbians compared to femme lesbians. In addition, research with OAEs show that they are more female typical in heterosexual women compared to bisexual women and lesbians (McFadden and Pasanen, 1998, 1999), 
Thus research does appear to support some role for prenatal androgens in women's sexual orientation, though there are conflicting and null findings. It seems highly unlikely that higher prenatal androgens are necessary for same-sex or bisexual sexual orientation to develop in women, and so it is unclear whether prenatal androgens contribute to some women's sexual orientation but not others and whether the strength of contribution might differ between women.

\section{PRENATAL HORMONES AND SEXUAL ORIENTATION IN MEN}

Studies on men's sexual orientation in response to clinical prenatal conditions or exposure are less common. However, men exposed to DES prenatally do not appear to differ in sexual orientation compared to controls (Kester et al., 1980), suggesting that exposure to masculinizing prenatal hormones in addition to typical male levels may not affect sexual orientation development. The 5-alpha-reductase deficiency has been studied internationally and is relatively common in some contexts, including localized areas within the Dominican Republic (ImperatoMcGinley et al., 1986). These XY individuals are generally reared as girls from birth, but at puberty $\mathrm{T}$ is sufficiently high to induce virilization, and most individuals, depending on cultural considerations, take on male roles (which generally includes partnering with women) in Papua New Guinea and the Dominican Republic (Imperato-McGinley et al., 1979; Imperato-McGinley et al., 1991). Because of the prevalence of the disorder, however, families may rear their children accordingly.

While digit ratios seem to show some evidence of prenatal masculinization in women with same-sex sexual orientations, evidence in men is mixed. Some studies point to prenatal masculinization in men (in opposite direction to the prenatal androgen hypothesis) such that men with same-sex sexual orientations exhibit lower (that is, more male-typical) digit ratios than men with opposite-sex sexual orientations (Robinson and Manning, 2000; Rahman and Wilson, 2003; Rahman, 2005a). Others have reported the converse, with higher digit ratios in men with samesex sexual orientations (McFadden and Shubel, 2002; Lippa, 2003). In contrast to women, there is no evidence that more masculine gay men exhibit masculinized digit ratios relative to more feminine gay men (Rahman and Wilson, 2003). There has been recent discussion about what this evidence suggests - that is, hyper- or hypomasculinization - and eth nic differences between populations have been suggested as one source of the differences between results. Even if (or especially if) these differences can be explained by ethnic differences, evidence that sexual orientation in men is associated with prenatal androgens is not supported by research using digit ratios as a putative marker of prenatal androgens. OAEs also have not supported the prenatal androgen hypothesis in men, though there has been slight suggestion of hypermasculinization when evoked OAEs are measured (McFadden and Pasanen, 1998, 1999).

\section{Adult Circulating Hormones and Sexual Orientation}

A common lay assumption holds that circulating androgens are associated with sexual orientation, with same-sex sexual orientations associated with higher $\mathrm{T}$ in women and lower $\mathrm{T}$ in men. Measurement of circulating androgens in adulthood is much more straightforward than direct or estimated measurement of prenatal hormones, but sampling issues are still important. If only certain subgroups of gay men and lesbians are willing and/or able to openly identify as gay, lesbian, queer, and/or same-sex oriented, and these groups do not represent all gay men and lesbians, then one could potentially be comparing a nonrepresentative group of gay men and lesbians to a potentially representative group of heterosexual counterparts.

There has been little evidence that circulating $\mathrm{T}$ is higher in women with a same-sex sexual orientation compared to women with an oppositesex sexual orientation. Instead, there is some evidence that self-identified butch lesbians have higher $\mathrm{T}$ than heterosexual women (Singh et al., 1999) or self-identified femme lesbians (Pearcey, Docherty, and Dabbs, 1996; Singh et al., 1999). This could be seen as suggestive that it is not sexual orientation per se that is associated with circulating $T$ but instead gender/sex. And unlike prenatal T, causality is generally unclear. Engaging in "male-typical" behaviors or behaviors intended to lead to a more "masculine" appearance over a long term could lead to higher androgens (for example, weight lifting: Linnamo et al., 2005). As with women, there has been little evidence that circulating $\mathrm{T}$ is higher in heterosexual men than in gay men. Unlike in women, there has been no evidence that $\mathrm{T}$ is higher in more masculine gay men compared to more feminine gay men.

Are Hormones Associated with Sexual Orientation in Women and Men

Findings provide little evidence associating prenatal $T$ with sexual orientation development in men. The evidence that does exist is generally contradictory. In women, research with clinical populations suggests that higher prenatal T might be associated with increased same-sex sexual orientations. As women are typically exposed to very low prenatal T, it may be that variations are large enough to affect neural development, whereas men's higher typical exposure to prenatal T (Nagamant ef al. 
1979) precludes effects on neural development unless exposure is varied dramatically. Evidence from women who are XY with CAIS who show typical female development and sexual orientation supports this, as they are exposed to considerably different levels of prenatal $\mathrm{T}$ (that is, none) compared to unaffected XY individuals. Still, the overall lack of consistent evidence should not be interpreted as suggesting that there are no prenatal or biological influences on sexual orientation.

Research does not support an association between adult circulating $T$ and sexual orientation but is suggestive that adult $T$ may be associated with gender/sex roles in women. It would be helpful to know whether more masculine heterosexual women show higher $\mathrm{T}$ than more feminineidentified counterparts. If so, that would confirm that it is gender subgroupings in women that are associated with circulating $\mathrm{T}$ more than sexual orientation per se. In contrast, $\mathrm{T}$ does show consistent associations with partnering, as I detail in this next section.

\section{Androgens and Partnering in Men}

Contrasting Single and Partnered Heterosexual Men's T

Research on partnering and hormones with North American humans has reliably shown that single heterosexual men have higher $\mathrm{T}$ than heterosexual men who are married (Booth and Dabbs, 1993; Mazur and Michalek, 1998; Gray et al., 2002) or in long-term relationships (Burnham et al., 2003; Gray, Campbell, et al., 2004; Gray, Chapman, et al., 2004). This research (see Gray and Campbell, this volume) represents an interdisciplinary endeavor, with anthropologists, sociologists, and psychologists involved, resulting in data from North American societies and beyond.

This interdisciplinary diversity is also matched by diversity of populations, as anthropologists have begun to conduct important research with international populations. These findings are less consistent, which is not surprising given that patterns of partnering can be culturally specific (see Gray and Campbell, this volume). In research conducted in Beijing, China, Gray, Yang, and Pope (2006) have found, for example, that married fathers have lower $\mathrm{T}$ than married nonfathers, suggesting that fatherhood may decrease $\mathrm{T}$ or men with lower $\mathrm{T}$ may be more likely to be fathers. International approaches provide for research that attends to the diversity of human experiences and patterns of affiliation. This literature is based on theoretical perspectives that focus on trade-offs in male mating effort. This work is also directly or loosely theoretically associated with the challenge hypothesis (Wingfield et al. 1990; Wingfield, this volume).
The challenge hypothesis (Wingfield et al., 1990) posits that androgens should be high around times of social challenge, with a focus on seasonal rhythms (for example, high $\mathrm{T}$ during the breeding season). Though originally proposed in relation to avian endocrinology, and receiving the majority of its empirical attention from bird researchers, the challenge hypothesis has been used fruitfully by researchers focusing on many other species (including humans: see Archer, 2006, for a review), as its authors intended.

\section{Testosterone Trade-off Framework}

Based on a synthesis of these studies, theories, and related bodies of literature, a framework for trade-offs associated with $\mathrm{T}$ and social behaviors has been posited (van Anders and Watson, 2006b). The testosterone trade-off framework hypothesizes a trade-off between high $\mathrm{T}$ and competitive behaviors/states, on one hand, and low $\mathrm{T}$ and bond maintenance behaviors/states, on the other hand. Competitive is associated with resource acquisition, including defense of a resource in response to a real or imagined threat. Bond maintenance is associated with developing intimate and/or caring social bonds with others, including partners, infants, friends, or family. This framework is conceptually related to the challenge hypothesis (Wingfield et al., 1990; Wingfield, this volume). One key difference between the testosterone trade-off framework and others is that the testosterone trade-off framework focuses on behavioral "intentions" as the key differentiation between competitive and bond maintenance behaviors as opposed to behavioral targets. For example, I hypothesize that infant defense is competitive behavior (defending a resource, that is, offspring) associated with higher $\mathrm{T}$ based on the testosterone trade-off framework, instead of positioning it as representative of low parental care as per the challenge hypothesis. Another difference is that the testosterone trade-off framework does not focus exclusively on males or on breeding and reproductive behaviors. Like other frameworks, the testosterone trade-off framework is useful for psychological studies because it queries and allows for state/trait effects. Trait effects need not be conceptualized as innately predetermined, and trait levels of $\mathrm{T}$ may not be inborn or innate. It is possible that some events along a developmental trajectory have led to both stable characteristic behaviors and T levels. Evidence does support a link between T and social behaviors in humans, with higher T associated with competitive behaviors/states and lower T associated with bond maintenance behaviors/states (for reviews, see Archer, 2006; van Anders and Watson, 2006b). 


\section{Contributions from Diversity to Partnering and Hormones}

When findings are reported in one group (for example, men), it is difficult to resist the idea that they occur only in that group (that is, men) and not in others (that is, women), turning no findings into null findings. The converse is the difficulty of resisting the urge to extend findings that are reported in one group (for example, men) to all groups (for example, women and men). Both could lead to hypothesis generation of an ultimate or proximate nature based on an incomplete foundation. As noted, research with international populations has allowed us to see how generalizable findings on partnering and hormones from North American heterosexual men are (see Gray and Campbell, this volume) and shows that the association is complex and sensitive to cultural idiosyncrasies. In this section, I detail the contributions from research with diverse North American populations. Research including diverse relationship styles or types in North America can help to expand the foundation for theorizing hormone-partnering associations, as I hope to show.

Considering Partnering and T with Sexual

Orientation and Gender/Sex

Previous studies (for example, Booth and Dabbs, 1993; Mazur and Michalek, 1998; Gray, Campbell, et al., 2004; Gray, Chapman, et al., 2004) have compared single heterosexual men with heterosexual men in long-term relationships. Based on testosterone trade-offs, theoretical considerations suggested comparing two groups using a competitive/bond maintenance distinction. In the context of partnering, competitive might refer to trying to find or attract partners, and bond maintenance might refer to trying to develop and maintain close intimate bonds with partners. I was thus interested in contrasting partnered with unpartnered people. Partnered included people in long-term, committed relationships with one person (for example, marriage, cohabitation, common-law, long-term relationships). Unpartnered included single people, people who were dating, and people in multiple relationships. This was because dating, by definition, denotes lower commitment to a partner and the possibility of having additional dating partners. As well, I included people in multiple relationships because they had the possibility of having additional partners. I hypothesized that unpartnered people would have higher $T$ than partnered people, because being unpartnered is akin to a competitive state, while being partnered is akin to a bond maintenance state.
A crucial issue relates to state/trait effects and whether $T$ decreases on entering a relationship (suggesting state effects) or lower $\mathrm{T}$ predicted the likelihood of entering a relationship (suggesting trait effects). A better understanding of causality in hormone-partnering associations would lead to more directed theorizing of mechanisms and functions. To address this, I included a longitudinal aspect whereby I could follow people's relationship status and $T$. If entering a relationship decreases $T$, researchers might look toward aspects of being partnered that could affect $\mathrm{T}$, including commitment, physical partner presence, or lifestyle, and look to possible adaptive functions of decreased $T$. If lower $T$ predicts entering relationships, researchers might look to preferences for partners with lower $\mathrm{T}$ or individual preferences for long-term relationships with lower $\mathrm{T}$ individuals.

Including nonheterosexual men and women and heterosexual women in addition to heterosexual men was valuable. Understanding the populations in which relationship status and $\mathrm{T}$ are associated could help direct us more effectively to hypotheses of mechanisms and function. For example, if the effect is only seen in heterosexual men, that would be suggestive that it occurs only in men with opposite-sex partners. This might direct us to question something about male-specific attributes of nervous systems and female partnering cues.

Our study (van Anders and Watson, 2006a) gathered a sample from the community, nearby universities, and the local Pride Parade. Participants were divided into heterosexual and nonheterosexual based on "Kinsey's questions" (Kinsey et al., 1948) of directed sexual fantasy and behavior. Heterosexual individuals scored exclusively or nearly exclusively on opposite-sex sexual fantasy and behavior. Nonheterosexual individuals scored exclusively or moderately on same-sex sexual fantasy and behavior. Thus, nonheterosexual is a better and more apt qualifier than gay or lesbian, since our participants were not exclusively oriented toward same-sex sexuality.

Our results replicated findings of higher $\mathrm{T}$ in single heterosexual men compared to heterosexual men in long-term relationships (for example, Gray, Campbell, et al., 2004; Gray, Chapman, et al., 2004), by comparing unpartnered versus partnered people (see Figure 15.1). That is, heterosexual unpartnered men had significantly higher $T$ than heterosexual partnered men. In contrast, nonheterosexual men's T did not differ as a function of partnered status, suggesting that the effect is not generalizable to all men. There was also no significant difference in $\mathrm{T}$ between partnered and unpartnered heterosexual women, suggesting that the of fect may not be generalizable to all heterosexual individuals. Unpartnered 
a

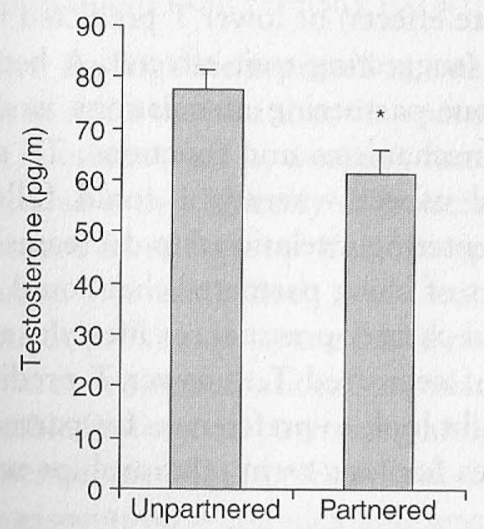

c

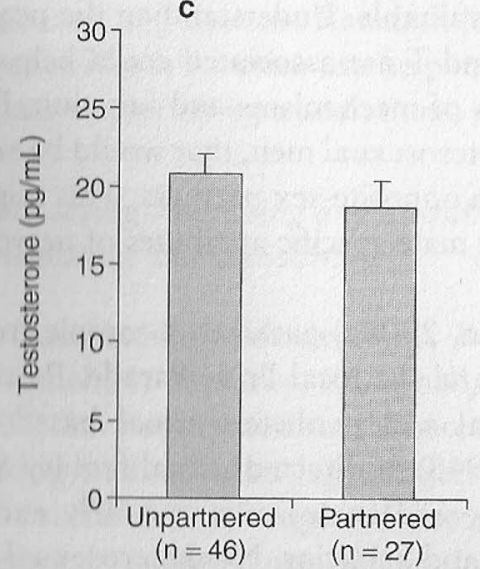

b
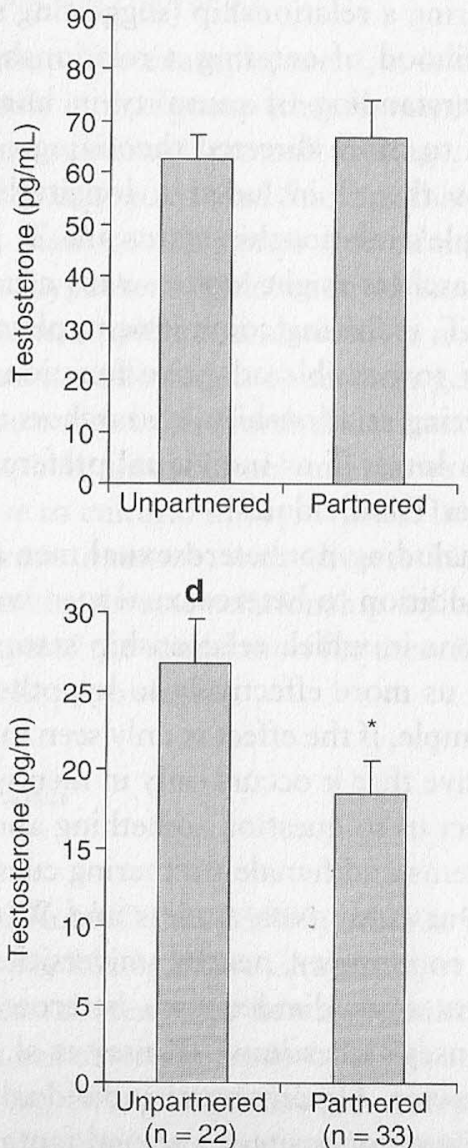

Figure 15.1 Mean baseline testosterone with standard error bars in partnered and unpartnered individuals in (a) heterosexual men; (b) nonheterosexual men; (c) heterosexual women; and (d) nonheterosexual women. * (asterisk) indicates a significant difference at $\alpha<0.05$. From van Anders and Watson, 2006a.

nonheterosexual women had significantly higher $T$ than partnered nonheterosexual women, duplicating heterosexual men. This suggests that the effect may occur in people who partner (or could partner) with women.

The longitudinal sample was small, and all participants showed changes in $T$ that were due to seasonality, with higher $T$ in the autumn (van Anders, Hampson, and Watson, 2006), Still, there was no evidence that T changes on entering a relationship, as individuals who entered a relationship showed variation in $\mathrm{T}$ over time that was similar to individuals who were in a relationship or unpartnered the entire time (van Anders and Watson, 2006a). Follow-up was 6 to 12 months after baseline testing, so it remains possible that longer time periods would result in a different pattern of findings.

Though there was no evidence of state effects in partnering and hormones, there was evidence that $T$ predicted entering a long-term relationship. At baseline, unpartnered individuals who would go on to be partnered had significantly lower $\mathrm{T}$ than unpartnered individuals who would remain unpartnered. This is suggestive that individuals with higher $T$ are less likely to enter long-term relationships and that the effect may be a trait one. This has led me to question whether we are examining associations between hormones and relationship status (that is, effects of current relationship status on $\mathrm{T}$ ) or hormones and relationship orientation (effects of $\mathrm{T}$ on later relationships) and whether there is such a thing as relationship orientation. There is evidence linking higher T levels with less need for long-term commitment (Cashdan, 1995), more frequent extramarital sex (Booth and Dabbs, 1993), more sexual partners (Bogaert and Fisher, 1995; Cashdan, 1995), and more interest in extramarital sexual partners (McIntyre et al., 2006), suggesting possible trait effects with relationship orientations associated with $\mathrm{T}$.

The findings from van Anders and Watson (2006a) can be compared with a retrospective study with very large sample sizes; Booth and Dabbs (1993) found that military men with higher $\mathrm{T}$ were less likely to have been married in the past and were more likely to have divorced. It could not be ascertained from their data whether these unmarried men had higher $\mathrm{T}$ because they were currently unmarried or because they were less likely to marry - that is, directionality of effect was unclear. In a longitudinal study with very large sample sizes, Mazur and Michalek (1998) compared T levels between consistently wed, unwed, and divorced military men. Consistently wed men had lower $\mathrm{T}$ than consistently unwed men, matching our findings. Divorced men exhibited similar $\mathrm{T}$ levels to the unmarried men. In addition, higher $\mathrm{T}$ was associated with the likelihood of divorcing, and $\mathrm{T}$ was transiently high around divorce. But if changes in relationship status did change $T$, then any increase in T occurring around divorce should still have been apparent later (and was not). Still, the data do demonstrate state effects from divorce on T. One difference between our results and Mazur and Michalek's (in addition to their much larger sample and time between initial and follow-up points) is that our study included a younger population who were not divoreing or remarying but instead were finding relationship 
partners. Likely, further longitudinal research will clarify our understanding of possible state effects.

The results in van Anders and Watson (2006a) suggest that relationship orientation may be associated with $\mathrm{T}$, as lower $\mathrm{T}$ individuals appear more likely to enter committed relationships. I can speculate that this might be associated with co-parenting, as long-term partnering often (though not always) is associated with childbearing and child rearing. If lower $T$ is associated with better bond maintenance behaviors, it would be advantageous for women (and possibly men) to pick low $\mathrm{T}$ (and high bond maintenance) partners for committed, long-term relationships. In support, lower $\mathrm{T}$ has been associated with better parental responsiveness in men (Storey et al., 2000; Fleming et al., 2002), better father-child relationships (Julian and McKenry, 1989), and time spent with spouses (Gray et al., 2002). As well, evidence suggests that fathers have lower T than nonfathers (see Gray and Campbell, this volume). It may be that low $\mathrm{T}$ individuals are more likely to be selected for long-term, committed relationships, and Roney et al. (2006) have found that low $\mathrm{T}$ men are viewed as more attractive for long-term relationships and more interested in infants. It may also be that high $\mathrm{T}$ individuals are more likely to select into short-term relationships, and evidence supports this as well (for example, Cashdan, 1995).

\section{Considering Partner Presence: Long-Distance Relationships}

Examining individuals who are single, in long-distance relationships, or in same-city relationships allows us to explore diversity in relationship types and further facilitates addressing the issue of relationship orientation versus status (van Anders and Watson, 2007). I hypothesized that if relationship orientation is associated with $\mathrm{T}$, then partnered individuals should have lower $\mathrm{T}$ than single individuals, regardless of the physical presence of their partners. If relationship status (that is, current affiliation) is associated with $\mathrm{T}$, then individuals in same-city relationships might differ from individuals in long-distance relationships, because physical partner presence (a salient cue to partner status) would differ. To test this, we recruited men and women from our university and the community.

Based on expectations from van Anders and Watson (2006a) where significant effects in women had only been seen with nonheterosexual women, it was surprising to find that women in same-city relationships had lower T than women who were single, even when sexual orientation was covaried. Women in long-distance relationships had $\mathrm{T}$ levels that were intermediate to single women and women in same-city relationships (see Figure 15.2). There was no evidence that other variables (for example, age, body mass index [BMI], sleep-wake cycles) accounted for the findings. Also, same-city and long-distance partnered women did not differ in any measured relationship variables (excepting physical contact with partner) including commitment, sexual attraction, sexual contact with nonpartners, relationship length, and plans to be with their partner forever. In van Anders and Watson (2006a), participants were not asked whether their relationships were long distance, and it is possible that some of them were, potentially obscuring any association between relationships and $\mathrm{T}$ in women. The findings described here suggest that relationship status and $\mathrm{T}$ are associated in women and that partner presence mediates this effect. Another possibility is that women in same-city relationships are more likely to behave in female-stereotyped ways or behaviors because their partners are present, and this gendered behavior affects $\mathrm{T}$. In possible support, self-identified femme partners have lower $\mathrm{T}$ than self-identified butch partners (Pearcey, Docherty, and Dabbs, 1996; Singh et al., 1999).

A different pattern of associations is apparent in men, such that men in same-city and long-distance relationships exhibit lower $\mathrm{T}$ than single men. This suggests that partnered men have lower T regardless of partner presence. These data are consistent with a relationship orientation explanation, since men in same-city and long-distance relationships would be subject to different partner cues. However, the similarity between commitment levels between men in same-city and long-distance relationships does not preclude the possibility that the state of commitment may lead to lower T. I do think, though, that commitment within a relationship may represent a separate variable from partnered status and may be more relevant to relationship orientation in some contexts. Interestingly, the results show that current partnered sexual activity could not be related to differences between partnered and single men's $\mathrm{T}$, since partnered men have lower $\mathrm{T}$ even when they differ in frequency of partnered sexual contact as partner presence necessitates. Investigations with long-distance and same-city partnered individuals have thus provided important insights, as has research with multiple partners, as I next discuss.

\section{Multiple Partners and Hormone-Partnering Associations}

According to testosterone trade-offs, people in multiple relationships should have higher T than people in coupled relationships, in part 


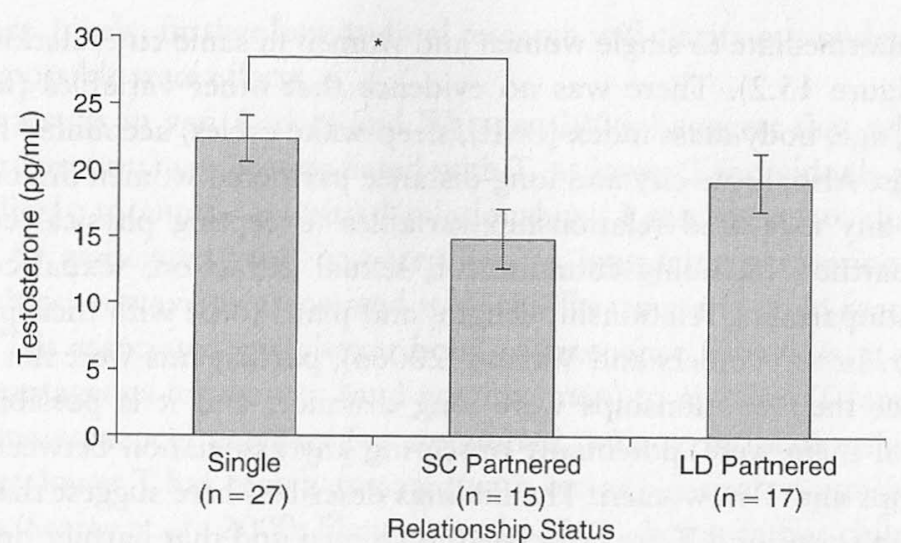

Relationship Status

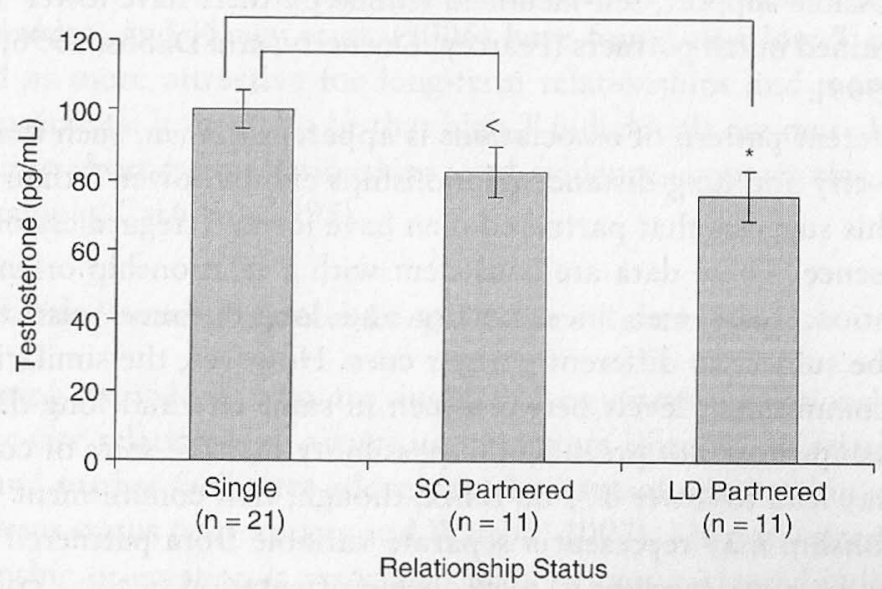

Figure 15.2 Mean testosterone levels by relationship status with standard error bars for (a) women (adjusted for age) and (b) men (adjusted for age and time of waking). SC Partnered, same city partnered; LD Partnered, longdistance partnered; * (asterisk) indicates a significant difference at $\mathrm{p}<0.05 ;<$ indicates a trend with $\mathrm{p}<0.10$. From van Anders and Watson, 2007.

because multiple relationships should afford the possibility and probability of still further relationships (a competitive-type state). To empirically address this, I studied polyamorous, monoamorous, and single people (van Anders, Hamilton, and Watson, 2007), Polyamory refers to "many loves" and characterizes multiple committed relationships or multipart ner relating, generally in a context of openness. Monoamorous individuals could be said to engage in monogamy (when they do), and polyamorous individuals could be said to engage in "polyfidelity," according to polyamory sources.

Despite the bonded nature of polyamorous relationships, I hypothesized that polyamorous individuals would exhibit higher $\mathrm{T}$ than monoamorous individuals, because polyamory should be associated with the possibility of new partners. In addition, I hypothesized that polyamorous individuals would have higher $\mathrm{T}$ than single individuals because polyamory is also associated with the probability of multiple and new partners in a way that being single is not.

I recruited participants from the community and polyamory groups. Polyamorous participants could be subdivided into people who currently had multiple partners (polyamorous) and people who were single or in monoamorous relationships but part of the polyamorous lifestyle (poly lifestyle). I also recruited people who were single or in monoamorous relationships. Previous research had shown that sexual orientation is relevant (van Anders and Watson, 2006a), so I covaried for sexual orientation, as there was not a large enough subsample of nonheterosexual people for analyses similar to the previous study.

Findings (see Figure 15.3) showed that monoamorously partnered men exhibit lower $\mathrm{T}$ than single, polyamorous, or poly lifestyle men. Interestingly, research with Swahili Kenyan men (Gray, 2003) supports this to some extent, as polygynously married men had higher $T$ than monogamously married men. However, monogamously married men did not have lower $\mathrm{T}$ than men who were single, perhaps reflecting cultural considerations. In van Anders, Hamilton, and Watson (2007), single men did have higher $\mathrm{T}$ than monoamorously partnered men. Also contrasting with Gray, we found that polyamorous men had higher $\mathrm{T}$ than single men, confirming all of our hypotheses. Thus, men with multiple partners or who are more likely to be looking for potential partners appear to be more likely to have higher $\mathrm{T}$, suggesting further a possible association between relationship orientation and $\mathrm{T}$ in men. Studies that are contextualized within varying cultures are likely to inform us further.

Findings in women also confirmed our hypotheses. Polyamorous women exhibited higher $\mathrm{T}$ than both monoamorously partnered and single women. As with the men, this remained true when we controlled for possibly relevant variables including sexual orientation. In contrast, however, single women did not exhibit higher T than monoamorously partnered women. The findings with women thus may point to an asso ciation between relationship status and $T$, such that only women eurrently with mulciple partaers exhibir higher T. 

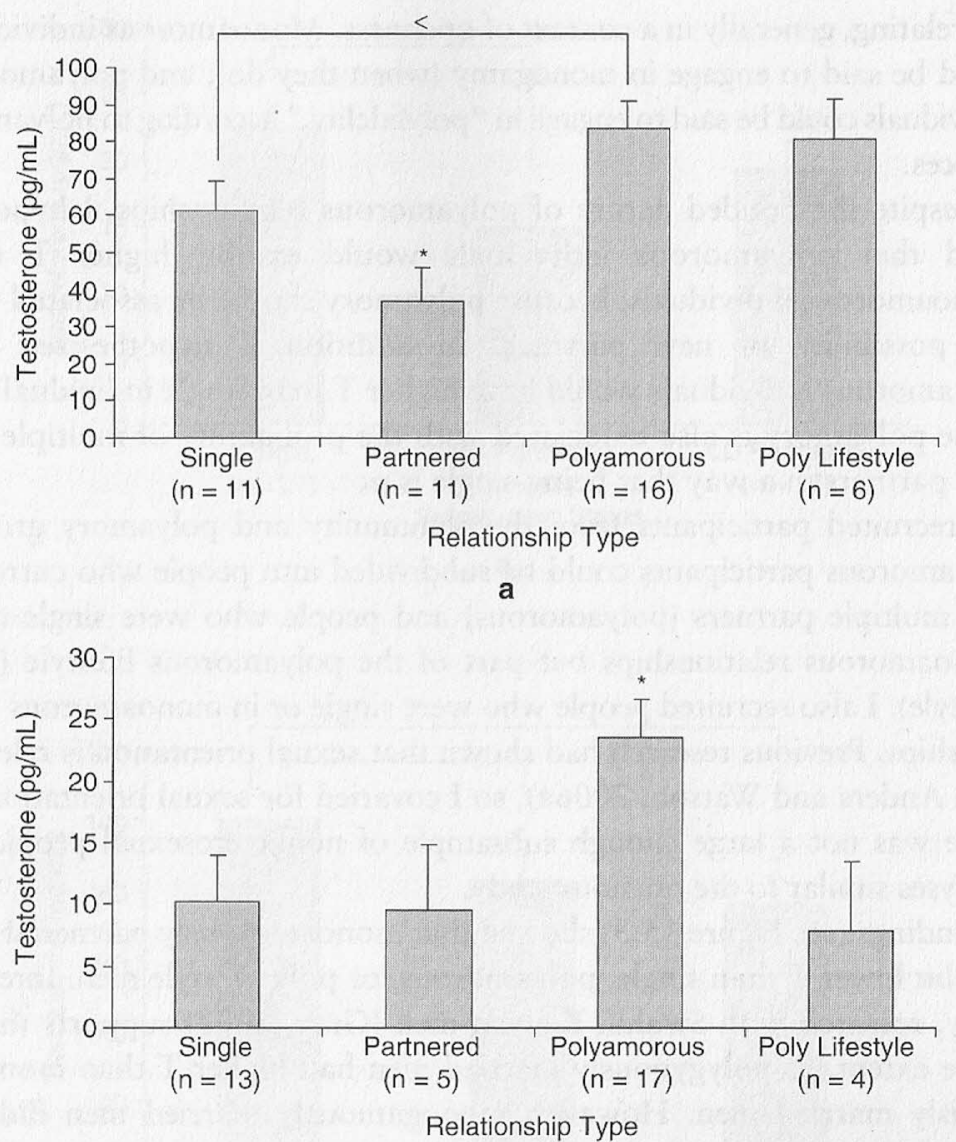

b

Figure 15.3 Mean testosterone levels and standard errors by relationship type, adjusted for age, sampling month, and sampling time for $(a)$ men and $(b)$

women. Poly lifestyle indicates participants not currently with multiple partners but identifying as having a poly approach to relationships, * (asterisk) indicates a significant difference from all other means at $p<0.05 ;<$ indicates a trend toward a significant difference from other means at $\mathrm{p}<0.10$. From van Anders, Hamilton, and Watson, 2007.

\section{Summary and Conclusion}

Findings on partnering and hormones show that monoamorously partnered men have lower $T$ than men who are in polygynous relationships (Gray, 2003), polyamorous relationships (van Anders, Hamilton, and Watson, 2007), or no relationships (van Anders and Watson, 2006a), Thes does not depend on physical pastner eves, as men in long-distance relationships have lower $\mathrm{T}$ than single men and similar $\mathrm{T}$ to men in samecity relationships (van Anders and Watson, 2007). Men with lower T are also more likely to enter relationships (van Anders and Watson, 2006a). These findings appear to apply only to heterosexual men (van Anders and Watson, 2006a), suggesting that partnering with women is somehow involved. There is evidence that women are more likely to pick lower $\mathrm{T}$ men for long-term partners (for example, Roney et al., 2006; see also Roney, this volume), so this may be an effect of female choice. However, men with higher $\mathrm{T}$ also appear less likely in a variety of ways to selfselect into long-term monoamorous relationships (for example, Cashdan, 1995).

The findings in heterosexual women are much less clear-cut and therefore less amenable to theorizing about ultimate explanations. So far, we know that partner presence is associated with lower $\mathrm{T}$ in women (van Anders and Watson, 2007); multiple partners are associated with higher $\mathrm{T}$ (van Anders, Hamilton, and Watson, 2007); single nonheterosexual women have higher $\mathrm{T}$ than partnered nonheterosexual women (van Anders and Watson, 2006a); and women with lower $\mathrm{T}$ may be more likely to enter committed relationships (van Anders and Watson, 2006a). These data conflict at times, and future studies should clarify what mediates and moderates associations between $\mathrm{T}$ and partnering in women and whether these associations are state, trait, or both.

Behavioral neuroendocrinology relies on the diversity of the natural world to deepen and develop understandings of hormone-behavior associations. It is not surprising, then, that this approach has been helpful in elucidating how partnering and androgens may be associated in humans. Attending to gender/sex, sexual orientation, and time has pointed my research in somewhat unexpected and certainly interesting directions. Though these may moderate the association between partnering and hormones, it appears that target partner's gender may also be implicated (van Anders and Watson, 2006a) and that T may influence partnering. Multitudes of questions remain. Are higher $\mathrm{T}$ individuals less interested in long-term relationships? Are women who are less interested in longterm partnering less likely to attend to cues of low $\mathrm{T}$ in their potential partners? Do female partners with lower $\mathrm{T}$ show higher parenting responsivity?

Findings are suggestive that relationship orientation more than status is associated with $\mathrm{T}$ in men, but perhaps the reverse in women. In a way, this contrasts with sexual orientation, as there appears to be little consistent evidence associating prenatal or circulating androgens with sexual orientation in men but some support for an association in women. Some evidence for relationshe orientation interpretations in men include that 
physical partner cues do not appear to be necessary for partnered men to exhibit lower T (van Anders and Watson, 2007); and lower T appears to predict entering committed relationships (van Anders and Watson, 2006a), staying wed (Mazur and Michalek, 1998), fewer sexual partners (Bogaert and Fisher, 1995), lower probability of extramarital sex (Booth and Dabbs, 1993), and more need for long-term commitment (Cashdan, 1995). Still, state effects are apparent in transient changes in $\mathrm{T}$ around divorce (Mazur and Michalek, 1998), as well as early stage love (Marazziti and Canale, 2004) and flirting (Roney, Mahler, and Maestripieri, 2003). Women in same-city relationships do show $T$ levels that are lower than single women and women in long-distance relationships (van Anders and Watson, 2007), suggesting that $\mathrm{T}$ may be associated with relationship status and orientation in women and that partner-related cues may affect women's $T$ in a statelike way. What are these cues? Do they stem from the partner, some physiological by-product in the women themselves, or from different lifestyles? Additional longitudinal studies and other studies that can address the state/trait issue are certainly warranted and would be helpful. The slash between state/trait does not indicate dichotomous thinking, and evidence will likely support various intermingled effects.

Research examining diverse relationship styles is likely to be informative. International research shows that having multiple female partners (for example, polygynous marriage) is associated with higher $\mathrm{T}$ in men (Gray, 2003), expanding our thinking about how and why partnering and $\mathrm{T}$ are associated. North American research also shows that multiple partners are associated with higher $\mathrm{T}$ in women and men (van Anders, Hamilton, and Watson, 2007) and that this extends to men who have a polyamorous approach to relationships but are not currently multipartnered. Would men who are oriented more toward multiple sexual contacts (for example, in swinging) as opposed to multiple committed sexual/romantic contacts, as in polyamory, show even higher T? Would women who are currently swingers have even higher T?

Exciting insights are likely to be gleaned from approaches that exam ine differences within broader groupings. Not all single individuals are cast from the same mold, and testable hypotheses abound for expectations of differences in $\mathrm{T}$ between subgroups of single, partnered, and dat ing individuals that may differ in temperament, experience, and interest. Diversity should be understood in its most inclusive form, including di. versity within major categorical divisions.

Researchers use various theoretical approaches to study partnering and hormones in humans; though this may be seen as detrimental, it is advantageous. Informative research is conducted under the aegis of the challenge hypothesis (Wingfield et al., 1990), in which high $\mathrm{T}$ in males is associated with more challenge behaviors (for example, aggression) and fewer behaviors indicating parental investment (for example, mate defense). Another perspective is the testosterone trade-off framework (van Anders and Watson, 2006b) in which high $\mathrm{T}$ in women and men is associated with more competitive behaviors (for example, infant defense, searching for partners) and fewer bond maintenance behaviors (for example, caring for partners or offspring). Additionally, researchers from various disciplines bring different viewpoints to similar questions, including state effects (how partnering affects hormones), trait effects (how hormones affect partnering), and reciprocal effects. The variety of perspectives allows for questioning and complementary insights.

Research that includes diverse human populations can benefit our understandings of the associations between androgens and partnering even for those not interested in diversity per se. Including these groups requires recognizing the value of diversity and inclusive research practices. Since individuals in these groups are often minority holders in power structures, it is crucial that research does not contribute to their marginalization or disempowerment. One reason is admittedly selfish: groups that are distrustful of science and scientists are unlikely to volunteer to participate in scientific studies. How could research continue in this case? Others are less so: the information gathered will hopefully further our understanding of human behavior and wonder at the complexity of human nature. Behavioral neuroendocrinologists who study geographically restricted species have come to appreciate conservation efforts both for environmental reasons and for the protection of future research and demonstrate convincingly that concerns about populations need not be divorced from science.

Attending to diversity has already provided us with further understandings of how androgens and partnering are associated in humans. Excitingly, including diversity has led to the generation of even more questions and testable hypotheses. By casting the net widely, researchers are increasingly likely to make sense of this hormone-partnering puzzle and its complicated pattern. It may be that the most improbable-seeming corners, the most seldom-viewed recesses, or the most overlooked spots hold the needed pieces. 